\title{
Critical Illness Related Corticosteroid Insufficiency in Trauma - A Review
}

\section{Mark L. Walker*}

Department of Surgery, Atlanta Medical Center, 303 Parkway Drive Atlanta, Georgia 30303, USA

\begin{abstract}
Critical IIIness Related Corticosteroid Insufficiency is an intense inflammatory condition associated with steroid tissue resistance. Although traditionally felt to be uncommon, it is being increasingly recognized in severely stressed patients with prolonged intensive care unit stays. Over the last decade the spectrum of $\mathrm{CIRCl}$ in trauma has been better defined. Trauma patients with CNS (brain and or spinal cord) injury, burns or blunt multisystem injury are at particular risk. The diagnosis is best established using a random cortisol level combined with an Adrenocorticotrophic Hormone (ACTH) stimulation test. A low cortisol level and or a low response to the ACTH stimulation test in the setting of refractory shock makes the diagnosis. Stress dose hydrocortisone therapy is essential and improves outcome. CIRCI should be suspected in any elderly trauma victim with a prolonged ICU stay that exhibits shock. Drugs known to inhibit cortisol synthesis (like etomidate) are probably best avoided in this trauma subset. $\mathrm{CIRCI}$ in trauma has a bimodal distribution. The first peak occurs early (within 48 hours) after injury and is associated with shock and the attendant inflammatory response. The second peak occurs a week or more into the hospital course. This peak is usually associated with sepsis. Inflammatory cytokines (particularly IL-6) are elevated during both peaks but their exact role in establishing the diagnosis remains unclear. Physicians continue to search for the Eucorticoid state that achieves a balance between the inflammation initiated by the injury and the anti-inflammatory response anchored by endogenous steroid production. The administration of exogenous steroids to achieve this balance is an approach that seems to hold promise.
\end{abstract}

Keywords: Critical Illness Related Corticosteroid Insufficiency CIRCI; Interleukin 6 - IL-6; Adrenocorticotrophic Hormone - ACTH; Central Nervous System - CNS

\section{Introduction}

Acute injury activates the hypothalamic-pituitary-adrenal axis. The HPA axis in combination with the autonomic nervous system and immune system produces the hormonal and cytokine milieu that allows the patient to respond to the initial insult [1]. This neuroendocrine and immune response involves a complex orchestration of hormones, cytokines, prostaglandins and other substances. An insufficient response increases the likelihood of significant morbidity and mortality. This review highlights our current understanding of critical illness corticosteroid insufficiency in the setting of trauma.

Critical Illness Corticosteroid Insufficiency is a pro-inflammatory state characterized by corticosteroid resistance [2]. One interesting theoretical concept proposed is that the level of adrenal response is inadequate for the given level of stress [3]. Put another way the inflammation initiated by the initial insult must be balanced by the anti-inflammatory effect of endogenous steroids. This has been referred to as the 'Eucorticoid' state [4]. When this balance is achieved, survival is likely. When inflammation proceeds unabated, end-organ injury and death becomes a major risk. The term Critical Illness Corticosteroid Insufficiency or CIRCI has replaced the older terms relative adrenal insufficiency and functional hypoadrenalism. When describing adrenal insufficiency in trauma we will use CIRCI to encompass all of these patients.

The incidence of CIRCI varies with the underlying illness, its' severity and duration [5]. Traditionally thought to be uncommon, CIRCI has been recognized in critically ill patients with sepsis, cirrhosis, coronary artery disease, trauma and many other conditions. The diagnostic criteria used to establish CIRCI will have an impact on the incidence [6-8].

\section{Background and clinical context}

Studies on the surgical stress response document a significant burden of inflammation related to open surgery. Increased cortisol levels are seen after major surgery [9]. Minimal access procedures produce a diminished inflammatory response and this observation supports why these procedures are often preferable in an elective setting [10]. Fast track adjuvants (including epidural anesthesia and steroids) can blunt the inflammatory response [11].

Burn injury studies in both children and adults highlight a large increase in circulating cortisol and catecholamines after significant injury $[12,13]$. In the setting of burn injury serum cortisol levels remain elevated for several months after injury. Past 100 days the levels begin to approach baseline (uninjured patients). Urinary catecholamines are also persistently elevated. These findings reflect the tremendous inflammatory and metabolic burden patients with large body surface area burns endure. HPA dysregulation has been noted in pediatric burn patients. Low baseline cortisol levels 72 hours after injury with an ACTH stimulation test that does not increase serum cortisol more than 9 micrograms per deciliter has been documented in a small cohort of pediatric burn patients [14].

In contrast, blunt trauma patients present a different pattern of cortisol response. Initially after injury the serum cortisol levels may be dramatically elevated only to return to a more normal level some 10 days out from injury [15]. Serum cortisol levels greater than $25 \mathrm{ug} / \mathrm{dl}$ are common after multi-system trauma [3]. The presence of hemorrhagic shock complicates this picture further as some of these patients may have low cortisol levels [16].

*Corresponding author: Mark L. Walker, Department of Surgery, Atlanta Medical Center, 303 Parkway Drive Atlanta, Georgia 30303, USA, E-mail: SurgHC1@aol.com

Received May 27, 2012; Accepted June 14, 2012; Published June 18, 2012

Citation: Walker ML (2012) Critical Illness Related Corticosteroid Insufficiency in Trauma - A Review. J Trauma Treat 1:139. doi:10.4172/2167-1222.1000139

Copyright: (c) 2012 Walker ML. This is an open-access article distributed under the terms of the Creative Commons Attribution License, which permits unrestricted use, distribution, and reproduction in any medium, provided the original author and source are credited. 
A trauma-induced catecholamine surge has been documented in a small cohort of bluntly injured patients. This increase in adrenaline has been linked to markers of tissue damage, coagulopathy and death [17]. The association between elevated adrenaline levels and poor outcome cannot be assumed to be causal. The findings from this small preliminary study raise the possibility that an over exuberant neuroendocrine responses can itself produce injury. Further work must be done to duplicate and expand these findings before firm conclusions can be reached.

Defining critical illness corticosteroid insufficiency biochemically has been problematic. Random cortisol levels may not accurately reflect adrenal function. The serum cortisol level reflects bound and free cortisol. $90 \%$ of cortisol is bound primarily to cortisol binding globulin [18]. The free cortisol is metabolically active. The bound cortisol is an inaccurate surrogate for the active moiety. In addition diurnal variation in cortisol levels can be misleading, making some researchers question the reliability of the random cortisol level [13]. In critical illness a random cortisol level of $<15 \mathrm{ug} / \mathrm{dl}$ has been proposed as an indication of CIRCI [18]. In severely stressed patients, a random cortisol level less than $25 \mathrm{ug} / \mathrm{dl}$ should raise the issue of CIRCI [3]. The ACTH stimulation test has been suggested as an effective way to detect inadequate adrenal response [19]. Hoen S et al. [20] noted a significant increase in interleukin 6 in trauma patients who were unable to respond to ACTH stimulation with a change in cortisol of greater than $9 \mathrm{ug} / \mathrm{dl}$. These early non-responders had greater volume requirements (colloid) and required more vasopressor support than responders. Fann SA et al. [21] have suggested that the need for vasopressor support and the need for intubation are predictive for the development of adrenal insufficiency in the setting of trauma. These are two indicators of profound shock and clinically should guide our thinking about this condition. Bernard F et al. [22] have shown that the incidence of CIRCI (for head injured patients) will vary based upon the criteria used to establish it. Strict biochemical criteria (a serum cortisol less than 10ug/ $\mathrm{dl}$ or a change in cortisol less than $9 \mathrm{ug} / \mathrm{dl}$ on ACTH testing) in the clinical context of refractory shock, establishes the diagnosis of CIRCI.

\section{Special trauma patient subsets at significant risk}

Elderly patients may have an impaired adrenal response due to aging [23]. These are patients with multiple co-morbidities and declining physiologic reserve. Prolonged critical illness itself has been linked to CIRCI in chronically ill medical patients [24]. Older patients with prolonged ICU stays are at risk $[25,26]$. Trauma among our seniors is increasing as many remain active and continue to drive in their later years. This is the population that we must carefully evaluate when the clinical situation raises the issue of CIRCI.

Patients with CNS injury; particularly closed head injury and or spinal cord injury may be at risk $[22,27,28]$. Direct trauma to hypothalamus or pituitary may occur. Increased Corticotrophin releasing hormone MRNA after brain fluid percussion injury in rats has been described [29]. In another animal study using a fluid percussion injury model, the administration of large doses of methylprednisolone was associated with pituitary apoptosis and increased mortality with a biochemical profile suggestive of CIRCI [30]. Chronic repeated head injury is also associated with pituitary injury [31]. Taken together this data suggests that blunt cerebral trauma activates the HPA axis, but in the setting of massive doses of methylprednisolone, hypothalamic and pituitary dysfunction (anatomic and physiologic) may occur.

Spinal cord injury may increase the risk of CIRCI. Lecamwasam published a case report of a patient with acute spinal cord injury who developed acute adrenal insufficiency after large dose glucocorticoid therapy [32]. Weant KA et al. [33] reported 2 patients with cervical cord injuries who developed acute adrenal insufficiency. Huang TS et al. [34] have documented impaired HPA axis responses to corticotrophin releasing hormone among 25 men with spinal cord injuries. All of these patients were more than 1 year out from the initial injury. 6 patients had no response to insulin hypoglycemia and minimal or no response to ACTH stimulation. Many cord injured patients are treated with pharmacologic dosages of methylprednisolone in an effort to minimize swelling. Chen's work cited above suggests that in the setting of cerebral trauma methylprednisolone may cause harm [30]. Supraphysiologic dosages of steroids may suppress endogenous production and disrupt the potential balance of a eucorticoid state. Very high cortisol levels have been documented in a small cohort of spinal cord injured patients [28]. All of these patients received pharmacologic methylprednisolone therapy to reduce cord edema. More than half of these patients were non-responders on ACTH testing.

As noted previously, patients who have sustained burns are a unique group. The prolonged inflammatory response seen in the setting of burns likely produces corticosteroid resistance. Prolonged elevations in serum cortisol have been clearly described. Disrupted cells in burn wound tissue may activate the conversion of cortisol to cortisone by enhancing 11B Hydoroxysteroid dehydrogenase [35].

This deactivation of cortisol may be another mechanism of corticosteroid resistance in burns.

Direct adrenal injury related to blunt trauma is an unlikely cause of CIRCI. In contrast, patients with bilateral adrenal hemorrhage in the setting of heparin-induced thrombocytopenia may be at increased risk [36].

Drugs may play a role in the development of CIRCI. Propofol, ketoconazole, rifampin and etomidate are some of the drugs that can impact adrenal function [5]. Of those mentioned, etomidate has generated the most concern. It is extremely useful because it does not produce cardiovascular dysfunction in patients about to be paralyzed and intubated. It causes adrenal suppression (for 24-48 hours) by inhibiting the 11B hydroxylase essential for steroid production [37]. Using this drug in the trauma setting may place patients at increased risk [38]. Although serum cortisol levels are lower [24] after etomidate is given, the short-lived nature of this suppression may not cause major problems for young trauma victims. For those older patients with multi-system trauma and for those with severe sepsis or septic shock, etomidate is best avoided.

A recent prospective double blind study evaluated intravenous hydrocortisone for 48 hours versus placebo in patients (two-thirds were trauma victims) who received etomidate. No difference in 28 day mortality or ICU stay was noted [39]. In this study patients who received steroids were able to wean from vasopressors more quickly. Overall hydrocortisone did not have an impact on outcome regarding etomidate induced adrenal suppression. Steroids cannot be recommended for this indication.

\section{CIRCI and the link to sepsis}

The animal and human data is compelling regarding the link of sepsis to hypothalamic-pituitary-adrenal axis activation and suppression. Michie HR et al. [40] have administered endotoxin to human volunteers and have documented a spike in TNF(tumor necrosis factor), ACTH and epinephrine early after the dose. The systemic and biochemical response can be muted by pretreatment with 
ibuprofen. This implies that the cyclooxygenase pathway is involved in mediating the effects of endotoxin. Endotoxin administered in rats, will produce the same ACTH spike as noted above. Administering an antibody to interleukin 6 abrogates this response almost completely [41]. High levels of inflammatory cytokines (particularly TNF) can directly inhibit adrenal cortisol synthesis [5]. Cationic peptides isolated from neutrophils called defensins also impact the HPA axis. These peptides can decrease cytokine production and reduce ACTH induced steroidogenesis [5]. The clinical impact of these observations in regard to the evolution and management of CIRCI remain uncertain.

\section{The clinical presentation and diagnostic work-up}

The major manifestation of CIRCI will be the presence of refractory shock. Adequate volume resuscitation and possibly vasopressor therapy has been started. Those patients who remain in shock in spite of these measures may have corticosteroid insufficiency. Rushing GD et al. [16] have documented low serum cortisol levels in 15 patients with hemorrhagic shock. There was a $13 \%$ mortality and prolonged hospital stay. If it is seen in the setting of sepsis, all of the systemic manifestations of infection may be present. Fever, tachycardia, tachypnea, altered mental state, with diminished urine output may be seen. If hemodynamic monitoring is available a hyperdynamic state is typical. Retrospective Corticus data suggests that mortality will be increased in those patients who do not respond to the ACTH stimulation test [42]. Although the major Corticus prospective study findings did not support a difference in outcome between responders and non-responders, time to shock reversal was shorter in those patients treated with steroids [43].

The biochemical work-up is the essential next step. A baseline cortisol level in the single digits in the setting of refractory shock is diagnostic. A positive ACTH stimulation test (with the change in cortisol of less than 9 micrograms per deciliter after the administration of 250 micrograms of ACTH) in the setting of refractory shock also makes the diagnosis. The Surviving Sepsis campaign (2008) addressed the issue of corticotrophin stimulation testing for patients in septic shock. These guidelines emphasize that clinicians should not wait for results of ACTH testing to administer steroids. Indeed these authors do not advise ACTH stimulation testing at all in the setting of septic shock [44]. Assessing glucocortioid tissue resistance remains an open question. T cells from burn-injured mice lose sensitivity to glucocorticoids [45].

Elevated serum cytokines in non-responders to the ACTH stimulation test have been documented in a small cohort of CIRCI patients [46]. Most of the cytokine increase was accounted for by the group with sepsis.

There is little published guidance regarding the use of serum cytokines to support the diagnosis of CIRCI. Electrolyte imbalance (low sodium or high potassium) is usually not present. Hypoglycemia may be present in profound adrenal insufficiency. Eosinophilia may be seen and should raise the issue of CIRCI when noted [26].

Imaging is usually not helpful in the management of these patients. CT scanning early on may reveal trauma to the adrenals. The absence or presence of adrenal trauma is not predictive of the future development of CIRCI [28]. In the setting of septic shock a recent study suggests that adrenal size (reflecting adrenal hyperplasia) might be associated with prognosis. The larger glands may be protective in the setting of septic shock [47]. Although this is a preliminary finding, it raises the question of whether extremely small glands are associated with CIRCI. There is one instance where imaging may be helpful: the presence of
Heparin-Induced Thrombocytopenia. Although uncommon, adrenal hemorrhage may be present and this can be documented potentially with CT [36]. MRI may be done to assess for spinal cord trauma or ligamentous injury but it has little role to play in the evaluation of trauma patients with CIRCI overall.

In summary, based on published literature, CIRCI has a bimodal distribution in the trauma setting. The first peak occurs early after trauma (within the first 24-48 hours). Persistent shock in the absence of occult hemorrhage and in spite of adequate volume resuscitation may be the initial clue. The second peak occurs later in the hospital course (usually by the second week). Sepsis is the initiating factor in this phase.

\section{Treatment}

Once the diagnosis of CIRCI has been made, the case for treatment should be based on improved outcome. Some authors have argued that non-response to the ACTH stimulation test need not be treated [14]. They also cite the prospective Corticus data showing no difference in outcome between responders and non-responders in the setting of septic shock [43]. Others who advocate no intervention, believe that CIRCI is similar to the Sick Euthyroid Syndrome.

In the setting of trauma, non-response to the ACTH stimulation test has been linked to increased vasopressor requirements, more volume needs and worse outcomes when compared to patients who respond (20,46). Guillomondeqgui et al., studied $82 \mathrm{ACTH}$ stimulation test non-responders. 66 were treated with steroids and 16 were not. Treatment reduced mortality by $50 \%$ [19]. Rivers noted improved survival and shorter time to shock reversal in a surgical cohort of patients with CIRCI treated with hydrocortisone [26]. In the retrospective Corticus study, 477 patients with severe sepsis or septic shock from 20 European ICU's were studied. An ACTH test was performed on the day of sepsis onset. Non-responders with any baseline cortisol level had a 1.38 odds ratio for mortality. Corticosteroid treatment improved outcome in both univariate and multivariate analysis [42]. Although the strength of some of this data can be challenged, taken together these studies and others suggest that clinical outcome may be improved when biochemically defined CIRCI is treated in the trauma setting. Randomized controlled trials regarding the management of CIRCI in trauma are currently not available. The recommendations regarding treatment below are based largely on observational studies that provides our best evidence at this time.

A stress dosage of hydrocortisone (200-300 mgs/day) for 10-14 day period is usually recommended. During this time, weaning from vasopressors should occur. For those patients with CIRCI and septic shock, mineralocorticoid therapy with fludrocortisone along with hydrocortisone may improve outcome [48]. Routine mineralocorticoid therapy as an adjunct does not seem to be warranted in most instances of CIRCI [3].

Steroid therapy is associated with significant adverse events. Steroid related GI tract ulcerations with bleeding, and superinfections are seen [43]. The ideal length of treatment is unknown. Several authors emphasize that abrupt cessation of steroids is associated with rebound inflammation that can seriously jeopardize recovery. For this reason they recommend a 3-5 day taper [3].

\section{Outcome}

Declining responsiveness to ACTH stimulation has been associated with multi-organ dysfunction and worse outcome in CIRCI [49]. This change in ACTH response reflects ongoing HPA dysfunction and not a 
Page 4 of 6

\begin{tabular}{|c|c|c|c|c|c|c|c|c|c|}
\hline Year of study & $\begin{array}{l}\text { Number of } \\
\text { patients }\end{array}$ & Age & ISS & Mean Cortisol & Stimulation Test & Rx'd Hydrocortisone & LOS & $\begin{array}{l}\text { Vent } \\
\text { days }\end{array}$ & Mortality Rate \\
\hline Offner et al. [15] & $\begin{array}{c}22 \\
13 \text { blunt }\end{array}$ & 30 & 34 & $30 \mathrm{mg} / \mathrm{dl}$ & no & no & & & $7 \%$ \\
\hline Hoen S et al. [20] & $\begin{array}{c}34 \\
34 \text { blunt }\end{array}$ & 34 & 29 & $19 \mathrm{mg} / \mathrm{dl}$ & yes & no & 49 & 12 & $14.7 \%$ \\
\hline Molina PE [1] & $\begin{array}{c}8 \\
5 \text { blunt }\end{array}$ & 47 & 28 & $7.5 \mathrm{mg} / \mathrm{dl}$ & & 8 & & & $12.5 \%$ \\
\hline Bernard F et al. [22] & $\begin{array}{c}113 \\
98 \text { blunt }\end{array}$ & 35 & 23 & $10.2 \mathrm{mg} / \mathrm{dl}$ & yes & yes & & 5 & \\
\hline Rushing GD et al. [16] & $\begin{array}{c}15 \\
10 \text { blunt }\end{array}$ & 50 & 23 & $15.8 \mathrm{mg} / \mathrm{dl}$ & no & & 27 & & $13 \%$ \\
\hline Fann SA et al. [21] & 63 & 35 & 22 & & & & & 19 & \\
\hline $\begin{array}{l}\text { GuillaMondequi OD et } \\
\text { al. [19] }\end{array}$ & 82 & 50 & 31 & $<20 \mathrm{mg} / \mathrm{dl}$ & yes & 66 & 23 & 11 & $25 \%$ \\
\hline Walker ML et al. [28] & $\begin{array}{c}70 \\
64 \text { blunt }\end{array}$ & 49 & 23 & $18 \mathrm{mg} / \mathrm{dl}$ & yes & 70 & 48 & 38 & $34 \%$ \\
\hline Roquilly A et al. [52] & 113 & 35 & 30 & 20 ug/dl & yes & 56 & & 1 & $10.7 \%$ \\
\hline Total & 511 & 41 & 27 & $17.2 \mathrm{mg} / \mathrm{dl}$ & & 313 & 37 & 17 & $16.7 \%$ \\
\hline
\end{tabular}

Abbreviations: ISS = Injury Severity Score LOS = Length of Stay Vent Days = Ventilator Days

Table 1: Collected Series of $\mathrm{CIRCl}$ in Blunt \& Penetrating Trauma.

shortcoming of the test itself. In this study, renal replacement therapy and thrombocytopenia were risk factors for a decline in delta cortisol. In another small study evaluating cytokine levels in CIRCI, elevated IL6, IL-10 and TNF levels were detected in non-responders with low (less than 9 micrograms per deciliter) delta cortisol [46]. C-reactive protein was higher in these patients and their overall outcome was worse when compared to patients with normal adrenal function or those with a low baseline cortisol levels. Even after head injured CIRCI patients have been weaned from ventilator support, elevated IL-6 levels have been documented [50]. Although causation is not proven by this data, it certainly suggests that HPA inhibition may occur in the setting of increased inflammatory cytokines and this may impact outcome.

Table 1 describes a summary of several clinical studies spanning a decade on CIRCI in blunt and penetrating trauma. 511 patients are included in this compilation. The average patient age was 41 years. The average ISS was 27 and the baseline cortisol was 17 micrograms per deciliter. Ventilator days were prolonged (average 17) as was length of stay (37 days). The mortality rate for this collected series was $17 \%$.

Recent burn center data for CIRCI, reveals an older patient cohort with a mean age of 51 years and large body surface area of injury (mean $33 \%)$. When compared to controls, CIRCI patients had a longer length of stay (mean 66 days vs. 35 days), prolonged ventilator days and a higher mortality (17\% vs. $2.5 \%$ ). Patients with more co-morbidities and inhalation injury were more likely to develop CIRCI [51].

\section{New Horizons}

A recent multi-center trial of continuous intravenous hydrocortisone for patients with traumatic brain injury was conducted [52]. 150 multiple trauma patients with cerebral injury were studied. The incidence of pneumonia and ventilator free days were the primary outcome variables. The pneumonia rate was dramatically reduced in the steroid group and ventilator free days were increased. These results were documented for both the CIRCI and non-CIRCI cohorts. The thinking is that the early administration of hydrocortisone blunted the systemic inflammatory response and changed the environment in a way that the patients' endogenous immune system could fight off potential infection. This takes us back to the concept of a Eucorticoid state. If the inflammatory response is balanced by endogenous or in this case exogenous steroids, survival and decreased morbidity will occur. Of course these results will have to be duplicated, but they remain provocative regarding what we may be able to accomplish to assist in recovery from severe injury.

\section{Conclusion}

Critical Illness Corticosteroid Insufficiency remains a significant risk to survival in the trauma setting. Although initially thought to be uncommon, CIRCI is being recognized more frequently with more rigorous testing. It has a bimodal presentation. Early on the severity of the insult and attendant inflammatory response may place the patient at risk. Later in the hospital course, sepsis is usually the initiating event. Serum cortisol monitoring and the ACTH stimulation test are critical studies that should be a part of the diagnostic work-up. Refractory shock should always raise this diagnosis. To avoid unnecessary morbidity and mortality this condition must be recognized quickly and rapidly treated. Stress dose hydrocortisone therapy is usually effective. The ideal duration of therapy remains unclear. CIRCI in trauma must be viewed as a condition of urgency.

\section{Acknowledgement}

Financial support from Surgical Health Collective and the use of the laboratory facilities at Surgical Health Collective are gratefully acknowledged. I want to express my gratitude to Ms. Amarachi Agada, for her assistance in manuscript preparation.

\section{References}

1. Molina PE (2005) Neurobiology of the stress response: contribution of the sympathetic nervous system to the neuroimmune axis in traumatic injury. Shock 24: 3-10.

2. Marik PE (2009) Critical illness-related corticosteroid insufficiency. Chest 135 181-193.

3. Marik PE, Pastores SM, Annane D, Meduri GU, Sprung CL, et al. (2008) Recommendations for the diagnosis and management of corticosteroid insufficiency in critically ill adult patients: consensus statements from an international task force by the American College of Critical Care Medicine. Crit Care Med 36: 1937-1949.

4. Burchard K (2001) A review of the adrenal cortex and severe inflammation: quest of the "eucorticoid" state. J Trauma 51: 800-814.

5. Marik PE, Zaloga GP (2002) Adrenal insufficiency in the critically ill: a new look at an old problem. Chest 122: 1784-1796. 
6. Asare K (2007) Diagnosis and treatment of adrenal insufficiency in the critically ill patient. Pharmacotherapy 27: 1512-1528.

7. Annane D, Maxime V, Ibrahim F, Alvarez JC, Abe E, et al. (2006) Diagnosis of adrenal insufficiency in severe sepsis and septic shock. Am J Respir Crit Care Med 174: 1319-1326.

8. de Jong MF, Beishuizen A, Spijkstra JJ, Groeneveld AB (2007) Relative adrenal insufficiency as a predictor of disease severity, mortality, and beneficial effects of corticosteroid treatment in septic shock. Crit Care Med 35: 1896-1903.

9. Kashiwabara M, Miyashita M, Nomura T, Makino H, Matsutani T, et al. (2007) Surgical trauma-induced adrenal insufficiency is associated with postoperative inflammatory responses. J Nihon Med Sch 74: 274-283.

10. Veenhof AA, Vlug MS, van der Pas MH, Sietses C, van der Peet DL, et al. (2012) Surgical stress response and postoperative immune function after laparoscopy or open surgery with fast track or standard perioperative care: a randomized trial. Ann Surg 255: 216-221.

11. Wilmore DW (2002) From Cuthbertson to fast-track surgery: 70 years of progress in reducing stress in surgical patients. Ann Surg 236: 643-648.

12. Norbury WB, Herndon DN, Branski LK, Chinkes DL, Jeschke MG (2008) Urinary cortisol and catecholamine excretion after burn injury in children. J Clin Endocrinol Metab 93: 1270-1275.

13. Vaughan GM, Becker RA, Allen JP, Goodwin CW Jr, Pruitt BA Jr, et al. (1982) Cortisol and corticotrophin in burned patients. J Trauma 22: 263-273.

14. Palmieri TL, Levine S, Schonfeld-Warden N, O'Mara MS, Greenhalgh DG (2006) Hypothalamic-pituitary-adrenal axis response to sustained stress after major burn injury in children. J Burn Care Res 27: 742-748.

15. Offner PJ, Moore EE, Ciesla D (2002) The adrenal response after severe trauma. Am J Surg 184: 649-653.

16. Rushing GD, Britt RC, Collins JN, Cole FJ, Weireter LJ, et al. (2006) Adrenal insufficiency in hemorrhagic shock. Am Surg 72: 552-554

17. Johansson PI, Stensballe J, Rasmussen LS, Ostrowski SR (2012) High circulating adrenaline levels at admission predict increased mortality after trauma. J Trauma Acute Care Surg 72: 428-436.

18. Cooper MS, Stewart PM (2003) Corticosteroid insufficiency in acutely ill patients. N Engl J Med 348: 727-734.

19. Guillamondegui OD, Gunter OL, Patel S, Fleming S, Cotton BA, et al. (2009) Acute adrenal insufficiency may affect outcome in the trauma patient. Am Surg 75: $287-290$

20. Hoen S, Asehnoune K, Brailly-Tabard S, Mazoit JX, Benhamou D, et al. (2002) Cortisol response to corticotropin stimulation in trauma patients: influence of hemorrhagic shock. Anesthesiology 97: 807-813.

21. Fann SA, Kosciusko RD, Yost MJ, Brizendine JB, Blevins WA, et al. (2007) The use of prognostic indicators in the development of a statistical model predictive for adrenal insufficiency in trauma patients. Am Surg 73: 210-214.

22. Bernard F, Outtrim J, Menon DK, Matta BF (2006) Incidence of adrenal insufficiency after severe traumatic brain injury varies according to definition used: clinical implications. $\mathrm{Br} \mathrm{J}$ Anaesth 96: 72-76.

23. Beale E, Zhu J, Belzberg $H$ (2002) Changes in serum cortisol with age in critically ill patients. Gerontology 48: 84-92.

24. Wu JY, Hsu SC, Ku SC, Ho CC, Yu CJ, et al. (2008) Adrenal insufficiency in prolonged critical illness. Crit Care 12: R65.

25. Barquist E, Kirton O (1997) Adrenal insufficiency in the surgical intensive care unit patient. J Trauma 42: 27-31.

26. Rivers EP, Gaspari M, Saad GA, Mlynarek M, Fath J, et al. (2001) Adrenal insufficiency in high-risk surgical ICU patients. Chest 119: 889-896.

27. Garcia-Zozaya IA (2006) Adrenal insufficiency in acute spinal cord injury. J Spinal Cord Med 29: 67-69.

28. Walker ML, Owen PS, Sampson C, Marshall J, Pounds T, et al. (2011) Incidence and outcomes of critical illness-related corticosteroid insufficiency in trauma patients. Am Surg 77: 579-585.

29. Grundy PL, Harbuz MS, Jessop DS, Lightman SL, Sharples PM (2001) The hypothalamo-pituitary-adrenal axis response to experimental traumatic brain injury. J Neurotrauma 18: 1373-1381.
30. Chen X, Zhang B, Chai Y, Dong B, Lei P, et al. (2011) Methylprednisolone exacerbates acute critical illness-related corticosteroid insufficiency associated with traumatic brain injury in rats. Brain Res 1382: 298-307.

31. Dubourg J, Messerer M (2011) Sports-related chronic repetitive head trauma as a cause of pituitary dysfunction. Neurosurg Focus 31: E2.

32. Lecamwasam HS, Baboolal HA, Dunn PF (2004) Acute adrenal insufficiency after large-dose glucocorticoids for spinal cord injury. Anesth Analg 99: 18131814.

33. Weant KA, Sasaki-Adams D, Kilpatrick M, Hadar EJ (2008) Relative adrena insufficiency in patients with acute spinal cord injury. Neurocrit Care 8: 53-56.

34. Huang TS, Wang YH, Lee SH, Lai JS (1998) Impaired hypothalamus-pituitaryadrenal axis in men with spinal cord injuries. Am J Phys Med Rehabil 77: 108112

35. Venkatesh B, Cohen J, Hickman I, Nisbet J, Thomas P, et al. (2007) Evidence of altered cortisol metabolism in critically ill patients: a prospective study. Intensive Care Med 33: 1746-1753.

36. Rosenberger LH, Smith PW, Sawyer RG, Hanks JB, Adams RB, et al. (2011) Bilateral adrenal hemorrhage: the unrecognized cause of hemodynamic collapse associated with heparin-induced thrombocytopenia. Crit Care Med 39 833-838.

37. Warner KJ, Cuschieri J, Jurkovich GJ, Bulger EM (2009) Single-dose etomidate for rapid sequence intubation may impact outcome after severe injury. J Trauma 67: 45-50.

38. Cotton BA, Guillamondegui OD, Fleming SB, Carpenter RO, Patel SH, et al (2008) Increased risk of adrenal insufficiency following etomidate exposure in critically injured patients. Arch Surg 143: 62-67.

39. Payen JF, Dupuis C, Trouve-Buisson T, Vinclair M, Broux C, et al. (2012) Corticosteroid after etomidate in critically ill patients: a randomized controlled trial. Crit Care Med 40: 29-35.

40. Michie HR, Manogue KR, Spriggs DR, Revhaug A, O'Dwyer S, et al. (1988) Detection of circulating tumor necrosis factor after endotoxin administration. $N$ Engl J Med 318: 1481-1486.

41. Perlstein RS, Whitnall MH, Abrams JS, Mougey EH, Neta R (1993) Synergistic roles of interleukin-6, interleukin-1, and tumor necrosis factor in the adrenocorticotropin response to bacterial lipopolysaccharide in vivo. Endocrinology 132: 946-952.

42. Lipiner-Friedman D, Sprung CL, Laterre PF, Weiss Y, Goodman SV, et al (2007) Adrenal function in sepsis: the retrospective Corticus cohort study. Crit Care Med 35: 1012-1018.

43. Sprung CL, Annane D, Keh D, Moreno R, Singer M, et al. (2008) Hydrocortisone therapy for patients with septic shock. N Engl J Med 358: 111-124.

44. Dellinger RP, Levy MM, Carlet JM, Bion J, Parker MM, et al. (2008) Surviving Sepsis Campaign: international guidelines for management of severe sepsis and septic shock: 2008. Crit Care Med 36: 296-327.

45. D'Elia M, Patenaude J, Dupras C, Bernier J (2010) T cells from burn-injured mice demonstrate a loss of sensitivity to glucocorticoids. Am J Physio Endocrinol Metab 299: E299-307.

46. Kwon YS, Suh GY, Jeon K, Park SY, Lim SY, et al. (2010) Serum cytokines and critical illness-related corticosteroid insufficiency. Intensive Care Med 36 1845-1851.

47. Jung B, Nougaret S, Chanques G, Mercier G, Cisse M, et al. (2011) The absence of adrenal gland enlargement during septic shock predicts mortality: a computed tomography study of 239 patients. Anesthesiology 115: 334-343.

48. Annane D, Sébille V, Charpentier C, Bollaert PE, François B, et al. (2002) Effect of treatment with low doses of hydrocortisone and fludrocortisone on mortality in patients with septic shock. JAMA 21: 862-871.

49. de Jong MF, Beishuizen A, van Schijndel RJ, Girbes AR, Groeneveld AB (2012) Risk factors and outcome of changes in adrenal response to ACTH in the course of critical illness. J Intensive Care Med 27: 37-44.

50. Dimopoulou I, Tsagarakis S, Kouyialis AT, Roussou P, Assithianakis G, e al (2004) Hypothalamic-pituitary-adrenal axis dysfunction in critically ill patients with traumatic brain injury: incidence, pathophysiology, and relationship to vasopressor dependence and peripheral interleukin-6 levels. Crit Care Med 32 404-408. 
Citation: Walker ML (2012) Critical Illness Related Corticosteroid Insufficiency in Trauma - A Review. J Trauma Treat 1:139. doi:10.4172/21671222.1000139

Page 6 of 6

51. Graves KK, Faraklas I, Cochran A (2012) Identification of risk factors associated with critical illness related corticosteroid insufficiency in burn patients. J Burn Care Res 33: 330-335
52. Roquilly A, Mahe PJ, Seguin P, Guitton C, Floch H, et al. (2011) Hydrocortisone therapy for patients with multiple trauma: the randomized controlled HYPOLYTE study. JAMA 305: 1201-1209. 